\title{
Implementing an intervention designed to enhance service user involvement in mental health care planning: a qualitative process evaluation
}

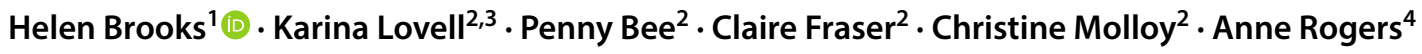

Received: 7 June 2018 / Accepted: 18 September 2018 / Published online: 28 September 2018

(c) The Author(s) 2018

\begin{abstract}
Purpose Shared decision-making (SDM) and the wider elements of intersecting professional and lay practices are seen as necessary components in the implementation of mental health interventions. A randomised controlled trial of a user- and carer-informed training package in the United Kingdom to enhance SDM in care planning in secondary mental health care settings showed no effect on patient-level outcomes. This paper reports on the parallel process evaluation to establish the influences on implementation at service user, carer, mental health professional and organisational levels.

Methods A longitudinal, qualitative process evaluation incorporating 134 semi-structured interviews with 54 mental health service users, carers and professionals was conducted. Interviews were undertaken at baseline and repeated at 6 and 12 months post-intervention. Interviews were digitally audio-recorded, transcribed verbatim and analysed thematically.

Results The process evaluation demonstrated that despite buy-in from those delivering care planning in mental health services, there was a failure of training to become embedded and normalised in local provision. This was due to a lack of organisational readiness to accept change combined with an underestimation and lack of investment in the amount and range of relational work required to successfully enact the intervention.

Conclusions Future aspirations of SDM enactment need to place the circumstances and everyday practices of stakeholders at the centre of implementation. Such studies should consider the historical and current context of health care relationships and include elements which seek to address these directly.
\end{abstract}

Keywords Care planning · Mental health · Process evaluation · User involvement · Qualitative · Implementation

\section{Introduction}

An enhanced focus internationally on incorporating user-led and recovery-oriented models of planning and management is predicated on the expectations of service users taking increased control of their lives [1]. This has been accompanied by the development of interventions that focus on service user and carer experience [2]. One particular area of change that has been articulated is care planning [1]. Evidence points to users feeling excluded, unsupported and distanced by mental health services and wanting more involvement in the care planning process [3, 4]. At policy level, enhanced involvement has been viewed as a means of improving the quality of care and promoting recovery $[5,6]$. However, there is considerable evidence that this does not occur easily and requires dedicated attention and action at a variety of levels to succeed $[4,7,8]$. 
In the United Kingdom, a care plan is defined as an agreement between a service user and their health professional designed to help them manage their everyday health [9]. These principles extend to the care planning approach (CPA) which is a national framework of care mandated for people with severe and enduring mental health problems such as schizophrenia and bipolar disorder [10, 11]. CPA involves an assessment of patient need, choices about care and support, consideration of family and financial matters and production of a care plan developed between professionals, the patient and their carer $[11,12]$. A recent systematic review identified literature relating the use of care planning processes in mental health services in a wide range of countries including the UK, USA, Australia and Sweden [4].

Shared decision-making (SDM) implicates the wider elements of the organisation and professional and lay practices in bringing about change. There is broad consensus among stakeholders about the value [13] of SDM but a complex and equivocal evidence base for its successful enactment [14]. A sustained policy emphasis on SDM has, therefore, yet to be universally translated into practice with limited evidence of how to ensure clinicians adopt and embed SDM routinely. From a user perspective, there is evidence that patients perceive participation (a central tenet of SDM) in multiple ways related to prior expectations of health care consultations and social position, suggesting a complexity extending beyond simply improving 'health literacy' and choice $[15,16]$.

SDM in mental health raises further challenges reflecting a context of practice in which patients are aware of the threat or actual containment and coercion $[17,18]$ which necessarily has a bearing on trust, ways of engaging and disclosure to professionals which is likely to impact on the quality of care planning relationships [19]. Additionally, user perspectives on interventions designed to more appropriately meet need have emphasised that they should be readily available and sensitive to community and domestic settings [20], and the context of everyday life which often lies out with the support provided by mental health professionals [21, 22].

\section{EQUIP intervention and trial}

The EQUIP intervention aimed to enhance service user and carer involvement in the care planning process through a 2-day training package targeted at all members of community mental health teams (CMHTs) responsible for providing care to people with serious mental health problems such as schizophrenia or bipolar disorder in ten NHS mental health trusts. Professionals in CMHTs come from a range of health and social care backgrounds, and can include psychiatrists, psychologists, community psychiatric nurses, social workers and occupational therapists [23].

The training was designed to introduce strategies to facilitate SDM in interactions with service users. The training was informed by interviews and focus groups with 51 mental health professionals [24], 42 service users [8] and 40 carers [7]. Data were synthesised at a 2-day event to design the training structure and content. This was attended by study co-applicants which included three service users and carer researchers. Nine service users and carers were recruited from either the study team (coapplicants) or from the study's advisory group who were then provided with a 4-day train the trainers course [25] to enable them to co-deliver the training in collaboration with academic trainers. Six of those who were trained went on to co-deliver the training course [26]. Table 1 contains more detail on the training.

The effectiveness of the training intervention was evaluated using a mixed design, including a cluster cohort sample, a cluster cross-sectional sample and process evaluation [27, 28]. The primary outcome for the trial was self-reported 'autonomy support'. Secondary outcomes included self-reported involvement in decisions, satisfaction with services, side effects of antipsychotic medication, well-being, recovery and hope, anxiety and depression scores, therapeutic alliance, quality of life and use of services [28].

Results from the randomised controlled trial found that the training intervention was well attended and received by staff. However, there was no significant difference in the primary outcome at 6 months as reported by service users in the control and intervention arms. Detailed findings from the cluster randomised controlled trial are reported elsewhere [28].

\section{Methods}

This manuscript reports on the nested qualitative process evaluation informed by implementation theory which aimed to explore the impact of the EQUIP training package to enhance user involvement in care planning.

Semi-structured interviews were undertaken by the lead author with service users, carers and mental health professionals from both control and intervention teams at three time points (baseline and 6 and 12 months post-intervention). Interviews were undertaken face-to-face or over the phone depending on participant preference. Face-to-face interviews were carried out either at participants' homes, on NHS or university premises or at a suitable community venue. The presentation of the methods and results is informed by the Consolidated Guidelines for the Reporting of Qualitative Data [29]. Baseline data have been presented elsewhere [30] and current analysis focuses on the implementation of training principles at 6 and 12-month post-intervention. 
Table 1 Further information on EQUIP training [26]

\begin{tabular}{|c|c|}
\hline Length of training & 2 days (starting at 9.30 and finishing at 16.30 ) \\
\hline Format of training & Face-to-face \\
\hline Location of training & Held at community mental health team bases or other NHS training venues or on university premises \\
\hline Day 1 training content & $\begin{array}{l}\text { Explanation of the EQUIP cluster randomised controlled trial (RCT) } \\
\text { Understanding the policy drivers relating to care planning and the experiences of service users and carers in receipt of } \\
\text { mental health services } \\
\text { Update on current evidence on service user and carer involved care planning } \\
\text { What does good care planning look like from multiple perspectives } \\
\text { Interactive exercises developing engagement and communication skills } \\
\text { Understanding care planning terms and processes }\end{array}$ \\
\hline Day 2 training content & $\begin{array}{l}\text { User-centred assessment } \\
\text { Exploring issues around 'risk' and 'safety' } \\
\text { Co-producing summary and formulation statements } \\
\text { Developing aspirational goals } \\
\text { What does shared decision-making look like } \\
\text { Thinking about user-involved implementation and reviewing of care planning }\end{array}$ \\
\hline Delivery & Role plays, interactive presentations, small group work, live examples of good practice \\
\hline Follow-up & $\begin{array}{l}\text { After the training, participants were emailed additional resources to complement learning and offered } 6 \mathrm{~h} \text { of clinical } \\
\text { supervision. Available here: http://research.bmh.manchester.ac.uk/equip }\end{array}$ \\
\hline Trainers & $\begin{array}{l}\text { Each training was run collaboratively by one of two academic researchers with a clinical background and one or two } \\
\text { service users and, where possible a carer }\end{array}$ \\
\hline $\begin{array}{l}\text { Service user and carer } \\
\text { trainer roles }\end{array}$ & $\begin{array}{l}\text { Group facilitators, sharing personal experiences of care planning and contribution to group discussions over the course } \\
\text { of the 2-day training }\end{array}$ \\
\hline
\end{tabular}

\section{Ethical approval}

Ethical approval was obtained from the National Research Ethics Committee North West-Lancaster [14/NW/0297].

\section{Participants}

54 participants (21 professionals, 29 service users and 4 carers, Table 2) were purposively sampled in relation to gender and geographical area from seven Mental Health Trusts (Table 3) involved in the trial. Information on participants retained at each follow-up point can be found in Table 4.

\section{Procedure}

Inclusion criteria were service users, carers or professionals from CMHT's included in the RCT. Service users were invited to take part through a written invitation, information sheet and consent to contact form. Staff members were approached through email. Interviews aimed to gather indepth data on the experience of utilising and receiving the EQUIP intervention and changes to practices over time (see Appendix 1 for an interview schedule). Interviews qualitatively explored any impact on outcomes identified as important within the RCT as well as giving participants the opportunity to discuss any additional outcomes.

\section{Data analysis}

Interviews lasting between 15 and 70 min undertaken between August 2014 and April 2017 were digitally audiorecorded and transcribed verbatim by an experienced, independent transcription company before being anonymised and allocated to a member of the research team for analysis. A thematic analysis was undertaken following the six stages outlined by Braun and Clarke by HB and AR [31] assisted by NVIVO. This involved reading and re-reading transcripts to ensure familiarisation with the data, generating initial codes, organising identified codes and developing overarching themes before reviewing and finalising themes [31].

$\mathrm{HB}$ and $\mathrm{AR}$ independently coded 12 interview transcripts ( 5 service users, 5 professionals and 2 carers) inductively and then met to develop a preliminary thematic framework. This was undertaken by extrapolating identified codes to a higher level of abstraction by examining similarities and differences between codes and considering relationships between codes [31]. The resultant framework was subsequently applied to the remaining transcripts by HB [32]. Further iterative modifications were made to the framework during this process which included the removal of duplicate codes, re-categorisation and the addition of new codes as new data were analysed. Analysis was supported through the use of the memo function on NVIVO to capture analytical decisions and an excel document which contained demographic information was used to contextualise the data. The framework was then discussed with the wider study team 
Table 2 Demographic information

\begin{tabular}{lr}
\hline Service users & \\
Male & 13 \\
Female & 16 \\
Trust 1: northwest of England & 12 \\
Trust 2: East Midlands & 5 \\
Trust 3: Northern England & 3 \\
Trust 5: northwest of England & 7 \\
Trust 4: Midlands & 2 \\
Intervention & 18 \\
Control & 11 \\
Total & 29 \\
Carers & \\
Male & 2 \\
Female & 2 \\
Trust 1: northwest of England & 1 \\
Trust 2: East Midlands & 1 \\
Trust 3: Northern England & 1 \\
Trust 4: Midlands & 1 \\
Intervention & 21 \\
Control & 1 \\
Total & 1 \\
Professionals & 2 \\
Male & 2 \\
Female & 3 \\
Trust 1: northwest of England $:$ Northern England & 1 \\
Trust 6: Northwest England & 1 \\
Trust 7: Northern England & 3 \\
Intervention & 4 \\
Total & \\
\hline & \\
\hline
\end{tabular}

to critically consider analytical interpretations and discuss any identified discrepancies. The resultant thematic framework was considered by authors to be reflective of participant data. Direct quotations along with thick descriptions of the data are included in the result section to promote transparency in the analytical process.

$\mathrm{HB}$ is a Lecturer in Psychological Sciences and a Health Service Researcher, PB a Professor in Mental Health Services Research, KL a Professor in Mental Health, CF and CM are Health Service Researchers and AR is a Professor of Health Systems Implementation. As such, no members of the research team had any prior relationships with study participants. This study forms part of an ongoing programme of research underpinned by a shared value in involving service users and carer in mental health services which are likely to have shaped interview schedules and the analysis process.

\section{Findings}

The three overarching themes and identified sub-themes are presented below.

\section{The sense and sense making of care planning training}

In terms of expectations, the views of service users, carers and professionals mainly coalesced in a shared understanding of the value and need for training to improve service user and carer involvement in care planning as current levels of involvement were considered insufficient. Inadequacies were sometimes attributed to other practitioners by professionals who described how such practitioners could become institutionalised into older ways of working oriented to traditional and paternalistic models of care.

We haven't really progressed very far in terms of being more person centred. We're still quite stuck in the medical model.

5022, professional, intervention

I'm not best pleased with it, because obviously I'm stuck on a CTO, but the care planning should involve
Table 3 Information relating to participating CMHTs

\begin{tabular}{lcc}
\hline Trust & $\begin{array}{l}\text { Number of professionals in partici- } \\
\text { pating CMHTs }\end{array}$ & $\begin{array}{l}\text { Number of service users } \\
\text { within participating } \\
\text { CMHTs }\end{array}$ \\
\hline Trust 1: northwest of England & 60 & 1355 \\
Trust 2: East Midlands & 26 & 310 \\
Trust 3: Northern England & 6 & 77 \\
Trust 4: Midlands & 25 & 638 \\
Trust 5: northwest of England & 16 & 424 \\
Trust 6: northwest of England & 18 & 278 \\
Trust 7: Northern England & 104 & 2318 \\
\hline
\end{tabular}


Table 4 Participants lost to follow-up

\begin{tabular}{lccll}
\hline Participant type & $\begin{array}{l}\text { Initial expression of inter- } \\
\text { est submitted }\end{array}$ & $\begin{array}{l}\text { Recruited at } \\
\text { baseline }\end{array}$ & $\begin{array}{l}\%(n) \text { of those recruited at baseline } \\
\text { followed up at } 6 \text { months }\end{array}$ & $\begin{array}{l}\%(n) \text { of those recruited } \\
\text { at baseline followed up at } \\
12 \text { months }\end{array}$ \\
\hline Service user & 47 & 29 & $90(26)$ & $83(24)$ \\
Carer & 9 & 4 & $50(2)$ & $25(1)$ \\
Professional & 31 & 21 & $76(16)$ & $52(11)$ \\
\hline
\end{tabular}

me more. I've been subjected to a care plan, rather than being involved in it.

6014, service user, control

The practice exemplars used in the training seemed to promote a sense of social comparison in which participants felt they were doing better than the examples provided in training. In retrospect, this may have led to a perception that they were doing relatively well and as a result the imperative to change their practice might have been diminished. The latter was supported by examining the changes in practice over the 12-month period which centred on changes in the use of terminology rather than the much harder to enact interactional aspects of SDM (Appendix 2).

I mean I did say too on the course that I felt...cos some of the examples they were giving we were horrified at, so I do think in a way you're working with a team here that is better than that.

\section{2, professional, intervention}

All stakeholder groups considered training was likely to work better for certain people including those in recovery, those whose first language was English and those that were new to services indicating a downward spiral of motivation for involvement. This consideration of those deserving or eligible for the new care planning approach may have limited the genericism of the application of the intervention in care planning practice.

I can't do it with every service user because some people, you know, they just, erm, they're either unwell or they've...they've, you know, they've got other issues. They don't want to do that. They're suspicious. I went to one yesterday, she's always got ongoing psychotic symptoms. She's very suspicious.

5008, professional, intervention

There's been times when I didn't really want any input from the support workers, just wanted to go me own way. Sometimes I've been so ill I just, it's been enough just to get through the day and I, I didn't really want a care plan. And I didn't want to be involved in it. Because I was so ill I just, I was just surviving really. 6002, service user, intervention
A minority of professional participants were resistant to the prospect of training and felt that there was no room for improvement in their practice and that training would not teach them anything new.

I didn't find the EQUIP training at all helpful because I didn't learn anything in it that we hadn't already been taught and that we didn't know.

5027, professional, intervention

I think we all thought it was a pain in the arse to be honest because...

...I think we all, have far too much work to do and the thought of giving up two full days, I think we all thought that, sort of, care management was our bread and butter.

5002, professional, intervention

However, most identified the uniqueness of the EQUIP training intervention in the context of a lack of awareness of any other care planning training available within the NHS. The co-delivery of training and the role of service users and carers in delivering the intervention were perceived to be a particular strength of the training.

It just makes it more pertinent. It just makes you think, I think about the person you're writing about really and that, you know, that these two people were saying, you know, that they'd had these care plans that, erm, they couldn't relate to at all and it...

...it did make you think, gosh, have I...have I written care plans like that, you know?

5002, professional, intervention

Despite identified benefits and ideological buy-in from most professionals initially, some were not confident from the outset that the training would result in demonstrable changes in every day care. The prevailing environment meant that in spite of co-developing aspirational goals with service users, a lack of resources would inhibit the addressing of service user needs in any meaningful way.

I think any change is really hard, and I think with all the pressures that we've got it's really hard to, to get 
it changed when...you're trying to catch up all the time anyway.

5009, professional, intervention

I mean I'm not trying to be rude but I don't think it sort of ever radically changes ones practice. It's not going to make a vast impact in terms of what the service users will experience day to day.

5019, professional, intervention

The broad training principles resonated with professionals and this sense making was seen as reinforcing the moral and ethical imperative to involve service users and carers in care planning (see Appendix 2). It ignited intentions to make decisions more collaboratively with service users and carers with participants describing how values of involvement had been introduced but eroded over time prior to training because of excessive workload and lack of resources. The training was considered successful in bringing this important component of practice back to the forefront of individual minds.

It [involving service users] should be part of what I do. And it's maybe been...just slipped away.

5003, professional, intervention

Because we don't have two days to think about practice, you know, you're literally firefighting and going from one job to another. So, like I say, even the sense of taking that time out was amazing and having the whole team there all feeling quite positive was just... was inspirational, it was really positive.

5022, professional, intervention

The team-based approach to training fostered collegiality by bringing staff together in a way they were generally not able to. Management support for the intervention also facilitated attendance. However, participants reported that despite this, psychiatrists within the team were reluctant to attend the training. This was reflected in a lack of attendance by psychiatrists across all teams included in the trial which may have impacted on the impact of the intervention.

The ideological commitment to training principles failed to translate into a new set of practices for most participants. The low expectations identified by professionals in relation to the potential lack of impact on actual care planning behaviours seemed to be a self-fulfilling prophecy. Followup interviews reported limited impact of the care planning training. Despite good intentions on the part of professionals after the initial training, they were not able to implement the changes that they had originally envisaged. Professionals acknowledged that even changes that they had been able to implement were likely to be so subtle that they may not be discernible to the service users they interacted with which was reflected in very limited numbers of service users noticing any changes over the 12-month period (Appendix 2).

I don't think it is more a dramatic change, maybe, you know, some of the, like, using terms like aspirational goals and working around them and maybe they might, you know, pick that out, I'm not too sure, so maybe some of the language, but I don't know if they [service users] would [notice any difference in practice], you know, really, because I think I've always, looked at... 5006, professional, intervention

So has anything changed in terms of your contact with mental health services over the last six months?

No, no, nothing at all.

No. Have you had any, um, care planning meetings or any care planning reviews?

No.

6015, service user, intervention

\section{The absence of the required relational work to enact the principles of SDM}

One reason for the lack of demonstrable impact of the EQUIP training was attributed to a lack of consideration given in the implementation plans to the significant relational work required to involve service users and carers in care planning. The context of mental health services which was considered fragmented and pressured made it difficult to undertake the requisite levels of relational work-that is the work that individuals need to develop and invest in negotiating relationships with others (Parker [33]). Such relational work included intra-professional relational work and the relational work between professionals and service users. Sufficient levels of the former were considered necessary to realise and optimise the latter.

We had the dis...such discontinuity with the medics...over the years. We've had no consistent medic for years and years and years. We've just had one part-time, erm, female consultant who left...to go to a different service. We've had nobody really else that's been that consistent and good.

5003, professional, intervention

...I have particularly noticed the last few weeks is how traumatic it can be for people to review their care plans. Particularly with a guy I met last week... And to look back at stuff that was written, you know, a few months ago and how much he has progressed from then, it brings back quite horrible memories for him. Yeah, it can be quite upsetting for service users to have to have a listen to that..... I don't know what the answer is to that. 


\section{0 professional, intervention}

Whilst some users reported positive relationships with mental health professionals, the data suggested long-standing difficulties in relationality often exacerbated by the absence of continuity and working on this over time. Service users described a lack of trust in the care dyad and professionals were frequently viewed as unreliable and unattuned to the every life, values, connections to others and the multifaceted needs of service users. The training did not appear to be sufficient to address this deep-rooted tension and enduring feature of user-professional relationships.

I've had CPNs in the past but they've been a bit of a waste of space, if you ask me. They didn't really offer that much support. They just sat there telling me what I should and shouldn't do. So when I've been offered them in the past, I've gone, no, you're alright. But because I have to have one because of the medication I'm on, I have to have a CPN so I don't really get a say in the matter.

5015, service user, intervention

I think it's hard really when you haven't had mental illness to know what the actual experience is for someone who has had the experience. So it's hard really. There's like a chasm, deep chasm between us - a growing canyon. They're on one side of it and we're on the other side of it.

6002 , service user, intervention

Stakeholders identified a range of individual level 'barriers' to implementation, including cynicism on the part of service users and professionals, periods of acute illness and attributions amongst professionals about levels of 'insight' and 'dependency' amongst service users. Professionals were concerned about involving carers in the process fearing that it would make the process more cumbersome and burdensome for them if carers' priorities were not aligned to those of service users and purported concerns about 'confidentiality'. Interestingly, these appeared to be largely unfounded when judged against the small number of instances where carer involvement had been successfully introduced and embedded because these concerns did not materialise in practice.

I think when carers maybe have their own conflicts with the person they care for in terms of what they need and how unwell they are, or, you know, what their care plan should be, we then have to sort of manage that tension listening to what the service user wants and the carer not being happy, you know, because they feel that there are other needs or something should be done differently, but it's not in line with what the service user wants.
5019, professional, intervention

\section{The failure of organisational readiness to support the workability of the intervention}

All stakeholder groups referred to contextual barriers to implementing user and carer-focussed care planning within mental health systems which directly overrode individual motivations and activity related to change. The EQUIP intervention was designed to work with existing organisational cultures, internal systems and processes. Stakeholders considered these organisational arrangements to be aligned historically to norms akin to a traditional medical model lacking sufficient patient orientation. Participants suggested a lack of fit between the intervention and health services due to limited value, and authentic and material commitment attached to service user and carer involvement by host organisations. Some targets introduced over the 12-month follow-up period were seemingly aligned to the values of the intervention but overall appeared to be superficial.

I don't know, it's hard to say that [if practice has changed] really, because it's the same pressures, I mean I haven't done a care plan since if that means anything. I hope it's made us all think a little bit more, which I think it does do, I think training does do that. It's just that it's still all the same pressures as to why our care plans maybe aren't as rich as they should be. 5019, professional, intervention

Participants considered a shift in language and engagement was required but this needed to be grounded in the meeting of expressed need. Holistic needs elicitation-a central feature of the SDM approach-was perceived to require articulation and nuanced discussion about what works for an individual. This required change in practice which did not integrate well with the pre-existing care planning language, templates, and systems that lacked flexibility and were unamenable to change. One imaginative team solution was to introduce electronic tablets for mental health professionals which were successful in facilitating collaborative working by reducing duplication. For needs elicitation to work, a level of continuity of care was required which was not possible in current services. Service users acknowledged this and often reported not seeing their named professional over the 12-month follow-up period.

It's very difficult seeing a different person every single time that doesn't know me from Adam, reads the last page of my notes because they haven't got time to sort of get a handle on me in the appointment process. So they only know a very little about me and go on sort of the last bit of data that's been put in the notes, and some of them are better than others, obviously. 
4027, service user, intervention

We do all have tablets now. We've got Internet access on the tablets and we can do some work with with clients while we're out. But... and hopefully it's coming in but it's not there yet, so our computerised notes system isn't on that tablet. So if that could be on it, then we can do the care plans and do things live and as we're there. Whereas at the moment, it's you'd have to type up, come back to the office, and then, you know, copy and paste and redo things.

5006, professional, intervention

Changes require time and consistent efforts for new ways of working to embed and routinize, and these may not have been realised in the 6-month follow-up period of the RCT.

The thing I remember is around aspirational statements and.... and goals, but obviously there's a lot more to it that because I haven't been able to use it as part of, you know, habit...it hasn't become habit forming and part of my practice, so, sort of, it's forgotten, sort of. 5006, professional, intervention

\section{Workability in context}

CMHTs were seen as the 'dumping ground' of mental health services and as a result workers were anxious about any additional workload, which they felt would adversely impact on staff sickness and attrition. Services were considered to be stretched by a lack of resources, increasing workloads and staff sickness and attrition. These contextual barriers seemingly overrode any action emanating from motivation for change instigated by the training. The intervention did not include a temporal plan in terms of a prospectus for change over time, further impeding the possibility of the actualisation of change.

It's the firefighting, you are literally moving from one thing to another and stealing bits of time from something else and you don't actually have that time to sit and think; time is the biggest barrier, so it's about making sure that it gets prioritised in terms of everything else that we have to do and that's always going to be a problem. My motivation to...to change things might slip.

5022, professional, intervention

I feel they're too hard pressed really. Coz, coz the senior support worker left, retired. And another support worker's left and they never replaced them. So there's more work for them to do. They are rushing, they've got too much to do in a day and there's too few support workers in my view.
6002, service user, intervention

Professionals such as psychiatric nurses considered that engagement and needs elicitation activities associated with service user involvement in care planning may be better facilitated by recovery workers, support workers and occupational therapists. This was because they felt they had more time to spend with service users to build the relationships required for shared decision-making to be realised in practice.

They've [support workers] got loads more time than you and they can actually get in and know people as well... I mean a lot of us had the skills to be able to come up with, with a [care] plan but we don't have the time. We don't have the time to go round somebody's house every two or three days and say, just walk to the end of the path with me. Let's stand here for ten minutes, let's...we don't. But support workers would be able to do that.

5003, professional, intervention.

My support workers that come round to the house, I think they're the ones. I mean they helped me when I had my last bad do. So they came in and, er, they were much better than the crisis team.

What was it, do you think about the support workers that made them good at - made them good at involving people?Well I think they did rely on their own experiences of life, the support workers. Um, and they come in every day and, er, you know, I think they came in for as long as was necessary.

6002, service user.

\section{Discussion}

This study was conducted as part of a wider process evaluation and ran parallel to the EQUIP RCT [28]. The lack of effect found in the RCT is consistent in most part with the findings from this study. The current study illuminated some of the reasons for this lack of effect which included a lack of organisational readiness and support for implementation and insufficient consideration and subsequent undertaking of the required relational work associated within the intervention. This was reinforced by the context encountered by mental health professionals which overrode initial enthusiasm and motivation for change following the training.

The work people need to undertake when implementing a new approach requires a context that is supportive of the new practice [34]. Professionals included in the process evaluation described how the training reinforced values about ethical and moral imperatives to involve service user and carers in treatment decisions, and fostered collegiality between 
colleagues through its team-based approach. In tension with this, however, professionals reported low expectations of changing actual practice following training and successfully predicted contextual barriers which negatively impacted on ability to implement user-centred care planning during the 12-month follow-up period. Some professionals reported being able to make changes to their care planning practice as a direct result of the EQUIP training (see Appendix 2) but acknowledged that because of the minor and subtle nature of these changes they may not have been discernible to service users. This was mirrored in the service user data. The current organisational focus on discharge and a lack of continuity of care within services more generally in the United Kingdom further diminished the likelihood of service users benefitting from these practice modifications. Work undertaken prior to the trial to identify potential implementation challenges to promote the implementation of training into practice [35] identified the need for managerial buyin to support the intervention. Whilst support was obtained for the training itself which facilitated attendance, further higher level support was not readily available to encourage the embedding of skills developed during the training which may have compounded the contextual barriers identified by participants.

This study shed light as to why the training intervention was ineffective in the short term. Our results suggest that the primary outcome of patient self-reported 'autonomy support' and attendant secondary outcomes insufficiently captured or reflected the ways in which people (users) planned for and enacted management on a daily basis or the way in which they interpreted how care planning change needed to be implemented systematically. The main trial outcome measure of shared decision-making traditionally rooted in a logic predicated on a notion of individualised choice aligned to a notion of an autonomous self represents a progressive view of user involvement. Participants in the current study coalesced in their shared value of service user and carer involvement in the care planning process. Nonetheless, it is limited by a tendency and objectification of standardized parameters and thus unable to capture all aspects which were important to users as far as planning for their care was concerned. Professional norms and values were overlain by fears and logistical factors operating in the workplace environment which impeded implementation. Our results show broader concerns of user engagement which lay out with the confines of shared decision-making in a traditional sense. The intervention failed to sufficiently consider these or the normal conditions into which it was being implemented which would have been required for the intervention to be workable in practice and to convert ideological buy-in into successful SDM in practice.

Baseline data demonstrated that care planning seemed insufficiently orientated to holistic needs assessment and that care plans had limited relevance to people's everyday lives [30]. Data from the current study added to these findings by identifying that stakeholders felt that professionals responsible for care planning did not have capacity to get to know service users well enough to undertake such activities optimally. As a result, stakeholders considered that alternative roles such recovery workers, support workers and occupational therapists may be best placed to undertake such activities.

Previous research shows how relationships together with environment, communication, trust and cultural competency contribute to the core of service users' experiences [36]. This study demonstrates that thorough consideration of the spectrum of relational work required to fully implement user centred care planning (e.g. intra-professional relational work in addition to user/professional relational work) was, with hindsight, an omission from the intervention design. Furthermore, the process evaluation raises concerns about whether the focus of the intervention was fully aligned with service user priorities, e.g. increased time and enhanced relationships with mental health professionals, [4] and whether the training was sufficient in challenging entrenched practices identified previously [37]. It may be that care planning focussed on managing mental health which is based on principles of connecting to others and activities that are valued in people's everyday life is likely to be a more effective and acceptable replacement to traditional care planning than trying to modify, through training, professional attitudes to user participation. Future studies should consider the historical context of health care relationships and include elements which seek to repair the relationships between service users and professionals often identified within the current data and elsewhere [7, 30, 35].

\section{Strengths and limitations}

The study draws its strength from the combination of its qualitative approach with the longitudinal design allowing for the in-depth exploration and nuanced understanding of the implementation issues associated with the intervention. Such methods enabled the predicted implementation barriers at baseline to be examined over the 12-month follow-up period and for the identification of unanticipated implementation factors that arose. Given the policy and practice mandates in this regard, many mental health organisations are seeking to improve service user and carer involvement in clinical decision-making and the findings from this study will support the development and implementation of interventions in this regard.

There were some limitations. First, participants selfselected themselves to be involved in the study and associated data may not be generalizable to other mental health stakeholders. Additionally, only those service users who 
were involved in the RCT were eligible for participation. Those service users who were acutely unwell or lacked professionally deemed insight to take part in the study were, therefore, ineligible for the process evaluation. Despite efforts to recruit carers, only four carer participants took part in the study. While carers' views were aligned to those of service users generally, given this low number carers' perspectives were under-represented. Additionally, the researcher undertaking the interviews was known by participants to be involved in EQUIP programme which may have impacted on the responses that participants gave.

\section{Conclusion}

Future aspirations of SDM enactment need to place the circumstances and everyday practices of stakeholders at the centre of implementation. Such studies should consider the historical and current context of health care relationships and include elements which seek to address these directly.

Acknowledgements This is a summary of independent research funded by the National Institute for Health Research (NIHR)'s Programme Grants for Applied Research Programme (Grant Reference number RP-PG-1210-12007). The views expressed are those of the author(s) and not necessarily those of the NHS, the NIHR or the Department of Health. The research team acknowledges the support of the National Institute of Health Research Clinical Research Network (NIHR CRN). The authors wish to thank all participants who took part in interviews for this research.

\section{Compliance with ethical standards}

Conflict of interest On behalf of all authors, the corresponding author states that there is no conflict of interest.

Open Access This article is distributed under the terms of the Creative Commons Attribution 4.0 International License (http://creativeco mmons.org/licenses/by/4.0/), which permits unrestricted use, distribution, and reproduction in any medium, provided you give appropriate credit to the original author(s) and the source, provide a link to the Creative Commons license, and indicate if changes were made.

\section{Appendix 1}

Example interview questions

Template of interview prompts for health professionals

What from your perspective are service users' perceptions of the user-led care planning (UCP)?

Who is most likely to engage with the UCP?

Who is most likely (in your experience) not to engage?

Who is UCP most suitable for?

In what circumstances would it be unsuitable?
Template of interview prompts for health professionals

Are there considerations of risk that need to be taken account of (for users, for others)?

What components of the UCP appear to promote positive engagement? (Discussion of biography, past activities, etc)

Clinical/health: what are the motivations for service users to engage with the UCP?

Social: do participants see the benefits of UCP and recognise an opportunity to develop their personal networks?

How do you think it does or could impact on the resources and networks of individuals?

What is the influence of current and previous engagement with activities on the uptake of UCP recommendations?

What are inhibiting or supporting characteristics of participants? (e.g. isolated, family pressures or diagnoses)

How did participants engage with ideas for UCP

How did the health professionals effectively engage participants and arrive at a plan?

Do participants relate their health problems with the intervention or the activities suggested?

What components of the UCP appear to resonate with participants in relation to managing their health?

How could UCP be improved?

Who is identified as potential support for engaging with new practices?

Specific questions relating to the utility for those in the intervention arm

Interview prompts for intervention patients

What does UCP mean to you?

Do you think UCP is relevant to you?

What parts of your last care planning meeting do you remember most? (Discussion of biography, past activities, etc)

Clinical/health: do you see any health benefits of UCP?

Social: do you see the social benefits of UCP?

What do you think helps or hinders you being involved in your care plan?

What do you think about your last care planning meeting?

Did you feel involved? How? Why

What are the facilitators/barriers to UCP?

Did you see the relevance of your UCP in relation to your health?

How could your involvement in care planning be improved?

What would you need to encourage you being involved in your care plan?

Who would help you do things for yourself?

Has UCP brought you in contact with any new people? (if so whom and what are the circumstances)

Do you think there are negative or risky aspects of UCP?

Specific questions relating to the identification of any changes in practice over time

\section{Appendix 2}

Examples of change identified within transcripts 


\section{2}

5003

5006
So I think, myself I've tried to cut out jargon... and make things more person specific. So rather than, develop coping mechanisms, I've put what they are. Like, so that the client knows exactly what we're talking about or I might put, develop ways to make you feel happier instead of, you know, terms like coping mechanisms

So that I've definitely written their care plans more in their language and the way they've spoken them rather than interpret them into a, kind of, nursey language

I get the sense that people are contacting families more and are aware about contacting families anyway that it's important to have input, not that we weren't doing it before but I think more so I think people are more....and I think people have been more creative about that, I'm certainly consulting families even if they can't get to a care plan I'm putting their views down, I'm ringing them and having a chat with them

I just like...the couple of care plans that I've done since I've been there [on the training]... I'm actually speaking to people again. It's made me talk to people again rather than just do them. And it's made me think about it from their point of view again without, erm, just kind of imposing what I think is best for them

I don't think it is more a dramatic change, maybe, you know, some of the, like, using terms like aspirational goals and working around them and maybe they might, you know, pick that out, I'm not too sure, so maybe some of the language, but I don't know if they [service users] would [notice any difference in practice], you know, really, because I think I've always, looked at...

I think definitely in terms of using the first person as if I was writing the care plan as the client. And using that to actually enhance genuine collaborative writing and... and the ownership of the care plan by the client. Erm, simplifying the language so there's less jargon and be more open to having both perspectives in the care plan

\section{8}

Just being very much more, sort of, patient-centred. That you get them to, you know, word for word tell you what you want them... what they want you... you know, get them to say it and then you write it down really as what they're saying and put it on the care plan and try and, you know, establish your goals with each thing that you do, erm, and how you're gonna do it and if you can, you know, get an aspirational goal off them

Since I've been on the EQUIP training, I do feel it is more, for me, I feel more confident in being more... a lot more collaborative and transparent about what we can and we can't do, erm, for people

5010

I think it probably has in that I'm possibly involving carers more... than I probably would've done. Erm, even even even though my background was from like crisis and you would often, erm, you know come across relatives or carers you know in my meetings there possibly didn't involve them as much you know in sort of care planning and stuff and I certainly do now

Maybe people will use the document a tiny bit more, you know try and use it instead of like get assumptions that someone doesn't want to get involved

I've started actually giving the documentation out to service users and saying, take it away, have a think about what your needs are in these areas and then get back to me and we'll make a plan together, so trying to make it something that's their document rather than ours

Being able to do the EQUIP training so early into my career really taught me what they were meant to be like and since then I feel that with $90 \%$ of my caseload I genuinely do follow all the learning and advice that I got from the training

I think it's fermented in my learning and understanding how important it is to use co-production and work with service users and learn from peers 
I've always thought that bringing carers, and bringing family members and friends, you know, if the services would like them to be involved, to bring them into the different interventions that you do. That's always been a priority for me, and certainly the training, kind of, hit that home. I think, in the community, it wasn't always possible, I think $80 \%$ of the time it's just because you just want to get it done as quickly as possible, and you knew that the carer, or the family member, wasn't going to be able to be there for another 3 weeks. I certainly thought, okay, actually, I don't have time to wait 3 weeks for this, because it's going to go out of date, I need to do it now. So, I just, kind of, tended to not be able to do it with the carer

I think it's probably similar to what I said to you when I spoke to you 6 months ago. I just try and use a care plan as a basis, begin it early on. I've tried to get better over the last 6 months about sending copies out, or providing copies by hand to people. Something that's probably more at the forefront of my mind most recently is giving GPs copies

I think in terms of carers being involved, I feel much more aware of that, just from having a lady on the course, you know, who was the carer, I did think that was a real...you know, hearing her perspective. Yeah, because sometimes just getting caught up and forgetting about that

\section{References}

1. Healthcare Commission (2008) The pathway to recovery: a review of NHS acute inpatient mental health services. http://www.ucl. ac.uk/core-resource-pack/resources/papers/healthcare_commi ssion_2008_The_pathway_to_recovery.pdf. Accessed 1 Feb 2018

2. Wallace $G$ et al (2016) Service user experiences of REFOCUS: a process evaluation of a pro-recovery complex intervention. Soc Psychiatry Psychiatr Epidemiol 51(9):1275-1284

3. Crawford MJ et al (2002) Systematic review of involving patients in the planning and development of health care. BMJ 325(7375):1263-1265

4. Bee P et al (2015) Systematic synthesis of barriers and facilitators to service user-led care planning. Br J Psychiatry 207(2):104-114

5. Department of Health (2000) The NHS Plan: a plan for investment, a plan for reform. http://webarchive.nationalarchives.gov. uk/20130124064356/http://www.dh.gov.uk/prod_consum_dh/

groups/dh_digitalassets/@dh/@en/@ps/documents/digitalasset/ dh_118522.pdf. Accessed 06 Jul 2018

6. Turner-Stokes L, Higgins B (2009) Advanced care planning. Concise guidance to good practice. Royal College of Physicians, London

7. Cree L et al (2015) Carers' experiences of involvement in care planning: a qualitative exploration of the facilitators and barriers to engagement with mental health services. BMC Psychiatry $15: 208$

8. Grundy AC et al (2016) Bringing meaning to user involvement in mental health care planning: a qualitative exploration of service user perspectives. J Psychiatr Ment Health Nurs 23(1):12-21

9. NHS Choices (2015) Your guide to care and support: care and support plans. http://www.nhs.uk/Conditions/social-care-andsupport-guide/Pages/care-plans.aspx. Accessed 06 Jul 2018

10. Department of Health (2018) Care programme approach. https:// www.nhs.uk/conditions/social-care-and-support/care-programmeapproach/. Accessed 21 Aug 2018

11. Department of Health (1999) Effective care co-ordination in mental health services: modernising the care programme approach-a policy booklet. http://webarchive.nationalarchives.gov.uk/+/http:// www.dh.gov.uk/en/Publicationsandstatistics/Publications/Publi cationsPolicyAndGuidance/DH_4009221. Accessed 06 Jul 2018

12. Department of Health (2008) Refocusing the care programme approach. Policy and positive practice guidance. http://webarchive .nationalarchives.gov.uk/20130124042407/http://www.dh.gov.uk/ prod_consum_dh/groups/dh_digitalassets/@dh/@en/documents/ digitalasset/dh_083649.pdf. Accessed 06 Jul 2018

13. Stewart M (2001) Towards a global definition of patient centred care- the patient should be the judge of patient centred care. BMJ 322(7284):444-445

14. Legare $\mathrm{F}$ et al (2010) Interventions for improving the adoption of shared decision making by healthcare professionals. Cochrane Database Syst Rev 12(5):CD006732

15. Protheroe J et al (2013) “Permission to participate?' A qualitative study of participation in patients from differing socio-economic backgrounds. J Health Psychol 18(8):1046-1055

16. Elwyn $\mathrm{G}$ et al (2008) Arduous implementation: does the Normalisation Process Model explain why it's so difficult to embed decision support technologies for patients in routine clinical practice. Implement Sci 3:57

17. Rogers A (1993) Coercion and voluntary admission — an examination of psychiatric-patient views. Behav Sci Law 11(3):259-267

18. Pescosolido BA, Gardner CB, Lubell KM (1998) How people get into mental health services: stories of choice, coercion and "muddling through" from "first-timers". Soc Sci Med 46(2):275-286

19. Edge D, Rogers A (2005) Dealing with it: Black Caribbean women's response to adversity and psychological distress associated with pregnancy, childbirth, and early motherhood. Soc Sci Med 61(1):15-25

20. Lamb J et al (2012) Access to mental health in primary care: a qualitative meta-synthesis of evidence from the experience of people from 'hard to reach' groups. Health 16(1):76-104

21. Biringer E et al (2016) Coping with mental health issues: subjective experiences of self-help and helpful contextual factors at the start of mental health treatment. J Ment Health 25(1):23-27

22. Walker $\mathrm{S}$ et al (2018) How do people with long-term mental health problems negotiate relationships with network members at times of crisis? Health Expect 21(1):336-346

23. Rethink mental illness. Community Mental Health Teams (CMHTs). Available at: https://www.rethink.org/diagnosis-treat ment/treatment-and-support/cmhts. Accessed 25 Sept 2018

24. Bee P et al (2015) Professional perspectives on service user and carer involvement in mental health care planning: a qualitative study. Int J Nurs Stud 52(12):1834-1845 
25. Fraser $\mathrm{C}$ et al (2017) EQUIP training the trainers: an evaluation of a training programme for service users and carers involved in training mental health professionals in user-involved care planning. J Psychiatr Ment Health Nurs 24(6):367-376

26. Grundy AC et al (2017) Evaluation of a co-delivered training package for community mental health professionals on service user- and carer-involved care planning. J Psychiatr Ment Health Nurs 24(6):358-366

27. Bower $\mathrm{P}$ et al (2015) A cluster randomised controlled trial and process evaluation of a training programme for mental health professionals to enhance user involvement in care planning in service users with severe mental health issues (EQUIP): study protocol for a randomised controlled trial. Trials 16:348

28. Lovell $\mathrm{K}$ et al (2018) Embedding shared decision-making in the care of patients with severe and enduring mental health problems: the EQUIP pragmatic cluster randomised trial. PLoS One 13(8): $\mathrm{e} 0201533$

29. Tong A, Sainsbury P, Craig J (2007) Consolidated criteria for reporting qualitative research (COREQ): a 32-item checklist for interviews and focus groups. Int $\mathbf{J}$ Qual Health Care 19(6):349-357

30. Brooks HL et al (2017) Is it time to abandon care planning in mental health services? A qualitative study exploring the views of professionals, service users and carers. Health Expect 21(3):59-605
31. Braun V, Clarke V (2006) Using thematic analysis in psychology. Qual Res Psychol 3(2):77-101

32. Richie J, Spencer L (1994) Qualitative data analysis for applied policy research. In: Bryman A, Burgess B (eds) Analysing qualitative data. Routledge, London

33. Parker VA (2002) Connecting relational work and workgroup context in caregiving organizations. J Appl Behav Sci 38(3):276-297

34. Lau $\mathrm{R}$ et al (2015) Achieving change in primary care-effectiveness of strategies for improving implementation of complex interventions: systematic review of reviews. BMJ Open 5:e009993

35. Brooks $\mathrm{H}$ et al (2015) Re-inventing care planning in mental health: stakeholder accounts of the imagined implementation of a user/ carer involved intervention. BMC Health Serv Res 15:490

36. Gilburt H, Rose D, Slade M (2008) The importance of relationships in mental health care: a qualitative study of service users' experiences of psychiatric hospital admission in the UK. BMC Health Serv Res 8:92

37. Brooks $\mathrm{H}$ et al (2017) Exploring the potential implementation of a tool to enhance shared decision making (SDM) in mental health services in the United Kingdom: a qualitative exploration of the views of service users, carers and professionals. Int J Ment Health Syst 11:42 